\title{
The Influence of Hydrogen Bonding on the Mesomorphic Properties of Side Chain Type Liquid Crystalline Polyurethanes with Rigid Moieties in the Polymer Backbone
}

\author{
Takashi MiHARA and Naoyuki KoIDE \\ Department of Chemistry, Faculty of Science, Science University of Tokyo, \\ 1-3 Kagurazaka, Shinjuku-ku, Tokyo 162, Japan \\ (Received July 24, 1996)
}

\begin{abstract}
In order to clarify the effect of the specific interaction of hydrogen bonding between urethane bonds (H-bonding) on the mesomorphic properties for side chain type liquid crystalline polyurethanes with a rigid moiety in the polymer backbone (rigid SLCPUs), side chain type polyurethane derivatives where hydrogen in urethane bonds were replaced with methyl groups were prepared by two synthetic routes, namely polycondensation and polymeric reaction. Their thermal properties were investigated by polarizing optical microscopy and differential scanning calorimetry (DSC) measurements. The original rigid SLCPUs exhibited mesomorphic properties, however, no mesomorphic properties were demonstrated in the polyurethane derivatives, regardless of the flexible spacer length. Therefore we concluded that the lateral interaction originating from H-bonding played an important role in the exhibition of mesomorphic properties for the rigid SLCPUs.
\end{abstract} KEY WORDS Side Chain Type Liquid Crystalline Polymer / Polyurethane / Hydrogen Bonding /

Thermal properties of side chain type liquid crystalline polymer (SLCP)s are greatly influenced by mesogenic groups, flexible spacer length introduced between the mesogenic group and polymer backbone, etc. We are interested in the specific interaction which could be organized between the amide group such as polyurethane and/or polyamide derivatives in the polymer backbone. We have already found that mesomorphic properties were exhibited for polyurethanes with a mesogenic group in the side chain. Furthermore, the relationship between the hydrogen bonding (H-bonding) formed by $\mathrm{NH}$ group and $\mathrm{C}=\mathrm{O}$ group of the urethane bonds in the polymer backbones and mesomorphic properties for side chain type liquid crystalline polyurethane (SLCPU)s was investigated by thermally controlled FT-IR. ${ }^{1-3}$ In order to clarify the effect of chemical structure on H-bonding in the polymer backbone, we synthesized SLCPUs with two types of polymer backbone as shown in Figure 1(A). One type was a polymer backbone containing a flexible alkyl chain (flexible SLCPU), the other one was a polymer backbone with a rigid moiety (rigid SLCPU).

In the case of the flexible SLCPU, a nematic phase was exhibited with a remarkable decrease in the strength of H-bonding. On the other hand, we were not able to clarify the relationship between mesomorphic properties and H-bonding for the rigid SLCPU.

Furthermore, in order to examine the influence of $\mathrm{H}$-bonding on the mesomorphic properties for SLCPUs, rigid SLCPUs with various kinds of mesogenic group were synthesized. The relationship between mesomorphic properties and $\mathrm{H}$-bonding for the rigid SLCPUs was also investigated by thermally controlled FT-IR. ${ }^{6}$ A small decrease in the strength of $\mathrm{H}$-bonding for the rigid SLCPUs was observed with increasing temperature. This tendency was dependent on the species of the mesogenic group. However the magnitude of change in the strength of the $\mathrm{H}$-bonding for the rigid SLCPUs was not remarkable compared with that of the flexible SLCPUs. A diagram of one of the rigid SLCPUs is shown in Figure 1(B).
In this study, in order to clarify the effect of H-bonding on the mesomorphic properties for the rigid SLCPUs, polyurethane derivatives where hydrogen atoms in the urethane bonds were replaced with methyl groups were synthesized by two synthetic routes, namely polycondensation and polymeric reaction. We examined the thermal properties of the polyurethane derivatives by polarizing optical microscopy and DSC measurements.

\section{EXPERIMENTAL}

\section{Characterization Techiniques}

${ }^{1} \mathrm{H}$ NMR was carried out with a JOEL JPN-PMX60 spectrometer using $\mathrm{CDCl}_{3}$ as the solvent. DSC measurements were conducted with a Mettler 3000 series. Polarizing optical microscopy was performed on a Nikon polarizing optical microscopy equipped with a Mettler FP80 controller and a FP82 hot stage.

Gel permeation chromatography (GPC) was carried out with a Tosoh HLC-8020 instrument using Chloroform as the eluent, equipped with four columns (TSK gel $\mathrm{G} 4000 \mathrm{H}_{\mathrm{HR}}, \mathrm{G} 3000 \mathrm{H}_{\mathrm{HR}}, \mathrm{G} 2000 \mathrm{H}_{\mathrm{HR}}$, and $\left.\mathrm{G} 2000 \mathrm{H}_{\mathrm{HR}}\right)$. The instrument was calibrated with a polystyrene standard.

Infrared spectroscopy were collected on a JOEL JIR-100 spectrometer. Spectra were collected at $4 \mathrm{~cm}^{-1}$ resolution. A minimum of 20 scans were signal averaged.
(A)

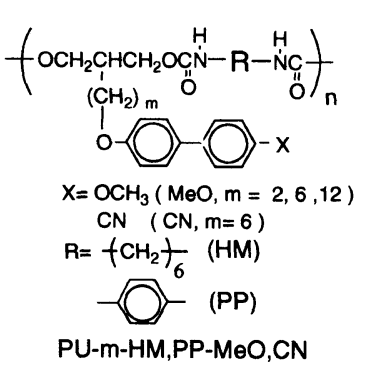

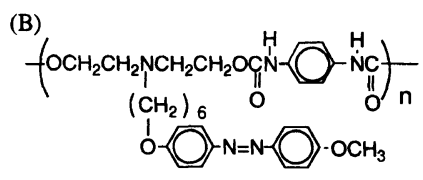

PU-MeOAZO
Figure 1. Structures of side chain type liquid crystalline polyurethanes. 


\section{Materials}

Synthesis and characterization of diol-monomers is described elsewhere. ${ }^{1}$ Polyurethane derivatives with methyl groups substituted for hydrogen atoms in the urethane bonds were synthesized according to Schemes 1 and $2 .^{4}$

$N, N^{\prime}$-Dimethyl-1,4-phenylenediamine (1). Lithium aluminium hydride $(4.4 \mathrm{~g}, 0.115 \mathrm{~mol})$ and $50 \mathrm{ml}$ of distilled tetrahydrofuran were placed in a dry reaction vessel. A solution of $p$-phenylenediisocyanate $\left(8.0 \mathrm{~g}, 5.0 \times 10^{-3}\right.$ mol) in tetrahydrofuran was added dropwise to the tetrahydrofuran dispersion of $\mathrm{LiAlH}_{4}(4.4 \mathrm{~g}, 0.115 \mathrm{~mol})$. After the reaction mixture was refluxed for 3 hours, the excess of $\mathrm{LiAlH}_{4}$ was decomposed with chilled water. The obtained reaction complex was hydrolyzed with $89 \mathrm{ml}$ of a $30 \% \mathrm{NaOH}$ aqueous solution. After evaporation of the tetrahydrofuran, the residue was refluxed for 2 hours. The black product was extracted with diethylether, and dried with anhydrous $\mathrm{MgSO}_{4}$. The diethylether was evaporated to dryness. The product was obtained in an $88 \%$ yield.

${ }^{1} \mathrm{H}$ NMR $\left(\mathrm{CDCl}_{3}\right): \delta 2.8\left(\mathrm{~s} ; 6 \mathrm{H}, \mathrm{NCH}_{3}\right), 3.3$ (s; $2 \mathrm{H}, \mathrm{NH}), 6.7$ (s; 4H, Ar-H)

IR (Nujol): $v 3342$ (NH), 1610, 1519 (Ar)

$N, N^{\prime}$-Dimethyl-1,4-phenylene Carbamoylchloride (2). After $150 \mathrm{ml}$ of ethylacetate was added dropwise to triphosgene $\left(25 \mathrm{~g}, 8.42 \times 10^{-2} \mathrm{~mol}\right)$ in a dry reaction vessel, a solution of $N, N^{\prime}$-dimethyl-1,4-phenylenediamine $(4.4 \mathrm{~g}$, $\left.3.17 \times 10^{-2} \mathrm{~mol}\right)$ in ethylacetate was added dropwise to the solution of triphosgene in ethylacetate. Following stirring at room temperature, a white precipitate was produced. The reaction mixture was refluxed at $55^{\circ} \mathrm{C}$ overnight. Ethylacetate solution was evaporated to dryness. The obtained solid was washed with $\mathrm{CCl}_{4}$. The product was obtained in an $85 \%$ yield.

${ }^{1} \mathrm{H}$ NMR $\left(\mathrm{CDCl}_{3}\right): \delta 3.4\left(\mathrm{~s} ; 6 \mathrm{H}, \mathrm{NC}_{3}\right), 7.3$ (s; 4H, $\operatorname{Ar}-\underline{\mathrm{H}})$

IR (Nujol): $v 1728(\mathrm{C}=\mathrm{O}), 1508$ (Ar)

Polyurethane Derivative (Polycondensation). Diolmonomer $(m=12)\left(0.35 \mathrm{~g}, 8.9 \times 10^{-4} \mathrm{~mol}\right)$ and carbamoylchloride (2) $\left(0.26 \mathrm{~g}, 1.0 \times 10^{-3} \mathrm{~mol}\right)$ were placed in a dry reaction vessel. Following the reaction of distilled $o$-dichlorobenzene $(5 \mathrm{ml})$, the reaction mixture was heated to $175^{\circ} \mathrm{C}$ for 96 hours. The polymer was obtained by the addition of $o$-dichlorobenzene solution to a large amount of methanol. The obtained polymer was washed with methanol. The polymer was obtained in a $67 \%$ yield.

PD-6-PP-MeO and $\mathrm{CN}$ were also synthesized according to the above described method. PD-6-PP-MeO and $\mathrm{CN}$ were obtained in $79 \%$ and $83 \%$ yield, respectively.

Polyurethane Derivatives (Polymeric Reaction). In an atmosphere of nitrogen, sodium hydride $(0.053 \mathrm{~g}, 2.2 \times$ $10^{-3} \mathrm{~mol}$ ) suspended in $2 \mathrm{ml}$ of DMF was stirred in a chilled reaction vessel. A DMF solution of polyurethane (PU-12-PP-MeO, $0.40 \mathrm{~g}, 6.6 \times 10^{-4} \mathrm{~mol}$ ) had been added to the suspension of sodium hydride via a cylinder. After the reaction mixture was stirred for $30 \mathrm{~min}$, methyliodide $\left(0.20 \mathrm{ml}, 3.2 \times 10^{-3} \mathrm{~mol}\right)$ was added dropwise to the mixture. Following stirring of the reaction mixture for 2 hours, excess sodium hydride was removed by filtration. The filtrate was concentrated and poured into a large amount of methanol. The obtained precipitate was washed with methanol. The polyurethane derivative was obtained in a $72 \%$ yield.

According to the above described synthetic method, PD-6-PP-MeO(PR) was also synthesized. PD-6-PP-MeO was obtained in a $93 \%$ yield.

$P D-M e O A z o$ (Polymeric Reaction). In an atmosphere of nitrogen, sodium hydride $\left(0.021 \mathrm{~g} 8.9 \times 10^{-4} \mathrm{~mol}\right)$ suspended in $2 \mathrm{ml}$ of DMF was stirred in a chilled reaction vessel. A DMF solution of polyurethane (PUMeOAzo) was added to the suspension of sodium hydride via a cylinder. After the reaction mixture was stirred for $30 \mathrm{~min}$, methyliodide $\left(0.1 \mathrm{ml} 8.94 \times 10^{-4} \mathrm{~mol}\right)$ was added dropwise to the mixture. After the reaction mixture had been stirred for 1 hour, it was evaporated to dryness. The residue was washed with water and methanol. The product (PD-MeOAzo) was obtained in a $93 \%$ yield.

\section{RESULTS AND DISCUSSION}

Polyurethane derivatives were synthesized by two synthetic routes. One route was polycondensation of bifunctional monomers and the other was polymeric reaction of the original SLCPU, as shown in Schemes 1 and 2 , respectively. The polymeric reaction was per-

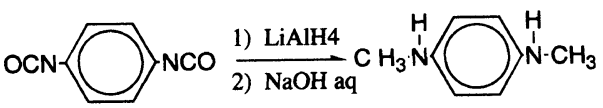

(1)

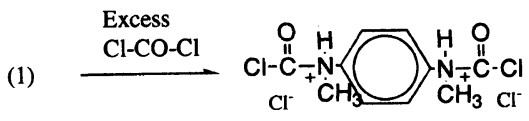

(2)

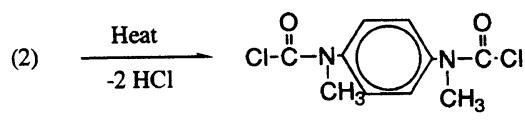

(3)
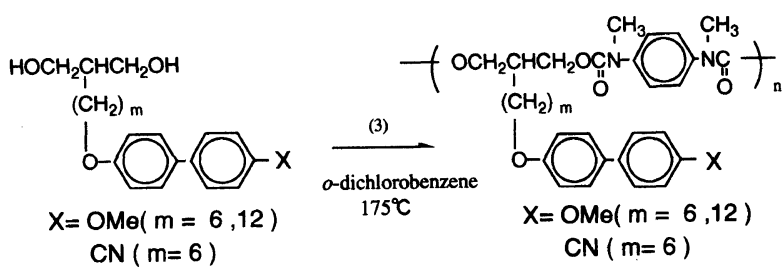

PD-m-PP-MeO,CN

Scheme 1. Synthesis of polyurethane derivatives (PD- $m$-PP-MeO, $\mathrm{CN})$.

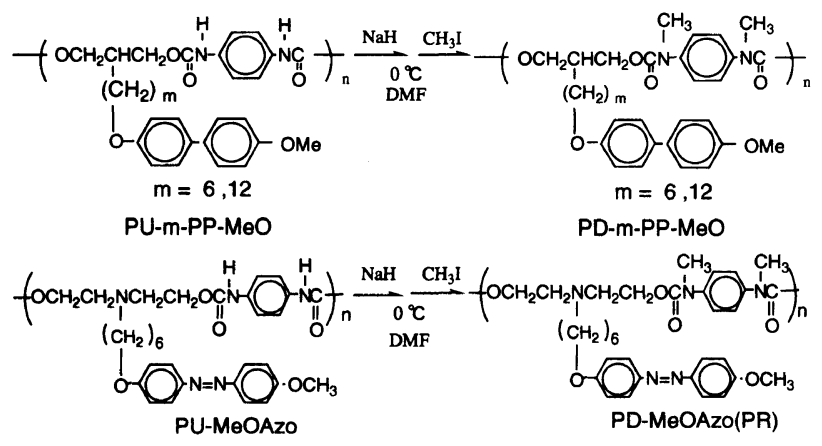

Scheme 2. Synthesis of polyurethane derivatives [PD- $m$-PP-MeO (PR) and PD-MeOAzo (PR)]. 


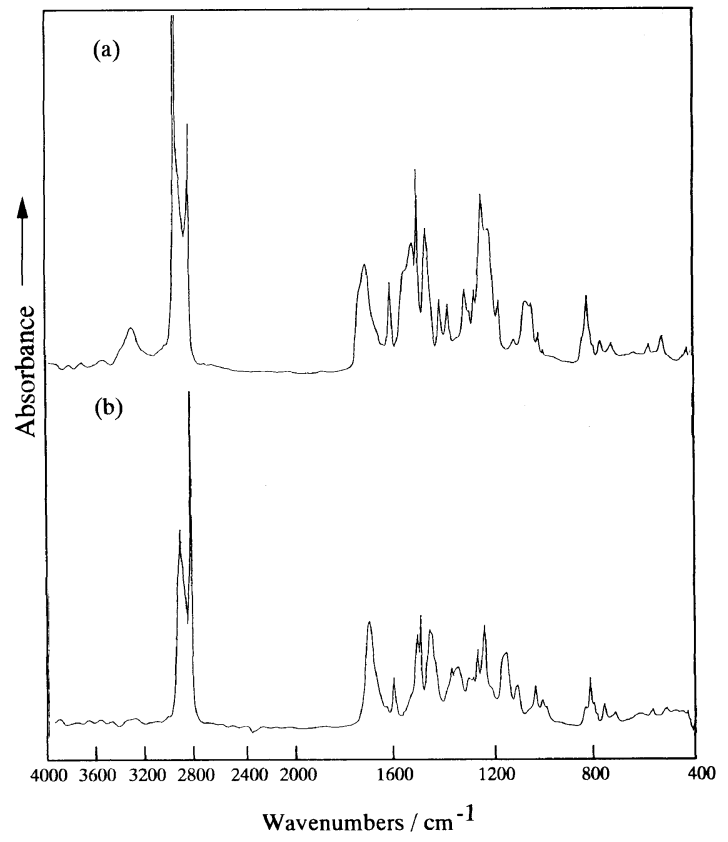

Figure 2. IR spectra of polymers [(a) PU-12-PP-MeO and (b) PD-12-PP-MeO(PR)]

formed at $0^{\circ} \mathrm{C}$, while the polycondensation was carried out at $175^{\circ} \mathrm{C}$. The PD- $m$-PP-MeO were prepared using both synthetic routes, whereas the PD-MeOAzo was synthesized only by polymeric reaction because thermal decomposition occurs during the polycondensation at high temperatures.

In the case of the PD- $m-\mathrm{PP}-\mathrm{MeO}$ and PD-6-PP-CN prepared by the polycondensation of bifunctional monomers, all hydrogen atoms in the urethane bonds in the polymer backbone were replaced with methyl groups. In the IR spectra of the PD-m-PP-MeO, and PD-6-PP-CN, no peak attributed to NH stretching vibration near $3300 \mathrm{~cm}^{-1}$ was observed. We confirmed that the PD- $m$-PP-MeO and PD-6-PP-CN where replacement of hydrogen atoms in the urethane bonds with methyl groups had occurred.

IR spectra of the PD synthesized by the polymeric reaction and the original SLCPU are shown in Figure 2. In the IR spectra of the polyurethane derivatives synthesized according to Scheme 2, the area of peak assigned to $\mathrm{NH}$ stretching vibration near $3300 \mathrm{~cm}^{-1}$ disappeared compared with that of the original SLCPU. This result showed that the polymeric reaction had caused hydrogen atoms in the urethane bonds in the polymer backbone to be replaced with methyl groups.

The thermal properties of original rigid SLCPUs are summarized in Table I. All the original rigid SLCPUs exhibited mesomorphic properties. With increasing spacer length for the PU-m-PP-MeO, a smectic phase was exhibited. This result was similar to the thermal dependence of the longer flexible spacer of conventional SLCPs and low molecular liquid crystal. ${ }^{5}$ Typical smectic and nematic phases were observed for the PU-MeOAzo.

The thermal properties of the polyurethane derivatives are summarized in Table II. In the polarized optical microscopy measurements, no birefringence was observed for all polyurethane derivatives with methyl groups substituted for hydrogen atoms of the urethane bonds in the polymer backbone. In DSC curves for the
Table I. Synthetic results and transition temperatures of side chain type polyurethanes

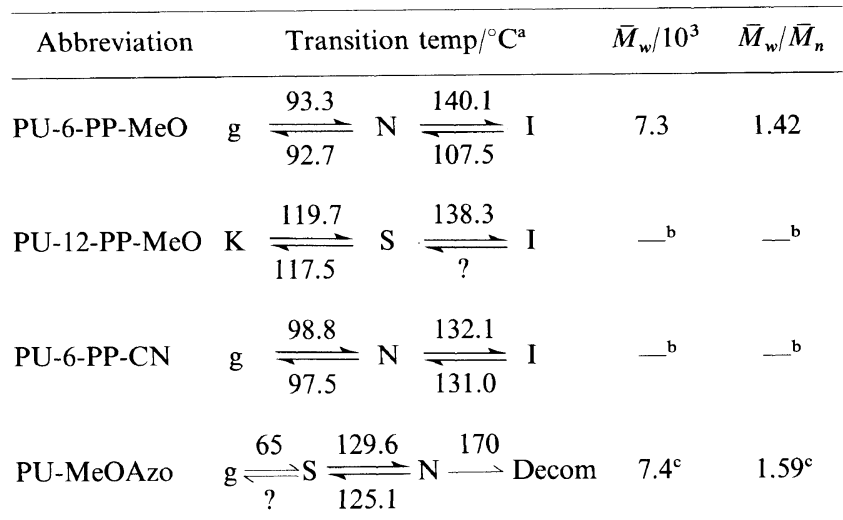

${ }^{\mathrm{a}}$ g, glassy; K, solid; S, smectic; N, nematic; I, isotropic. ${ }^{\mathrm{b}}$ Insoluble in chloroform. ${ }^{\mathrm{c}}$ Partially soluble in chloroform.

Table II. Synthetic results and transition temperatures of polyurethane derivatives

\begin{tabular}{|c|c|c|c|c|c|}
\hline $\begin{array}{l}\text { Abbreviation of } \\
\text { polyurethane derivative }^{a}\end{array}$ & \multicolumn{3}{|c|}{ Transition temp $/{ }^{\circ} \mathrm{C}^{\mathrm{b}}$} & $\bar{M}_{w} / 10^{3}$ & $\bar{M}_{w} / \bar{M}_{n}$ \\
\hline \multirow{2}{*}{ PD-6-PP-MeO } & \multirow[b]{2}{*}{$\mathrm{g}$} & 58.3 & \multirow{2}{*}{ I } & \multirow{2}{*}{6.8} & \multirow{2}{*}{1.12} \\
\hline & & $?$ & & & \\
\hline \multirow{2}{*}{ PD-6-PP-MeO(PR) } & \multirow[b]{2}{*}{$\mathrm{g}$} & $?$ & \multirow{2}{*}{ I } & \multirow{2}{*}{5.7} & \multirow{2}{*}{1.16} \\
\hline & & $?$ & & & \\
\hline \multirow{2}{*}{ PD-12-PP-MeO } & \multirow[b]{2}{*}{$\mathrm{g}$} & 61.9 & \multirow{2}{*}{ I } & \multirow{2}{*}{6.8} & \multirow{2}{*}{1.08} \\
\hline & & 57.7 & & & \\
\hline \multirow{2}{*}{ PD-12-PP-MeO(PR) } & \multirow{2}{*}{ g } & 68.0 & \multirow{2}{*}{ I } & \multirow{2}{*}{9.6} & \multirow{2}{*}{1.27} \\
\hline & & $?$ & & & \\
\hline \multirow{2}{*}{ PD-6-PP-CN } & \multirow{2}{*}{$\mathrm{g}$} & $?$ & \multirow{2}{*}{ I } & \multirow{2}{*}{5.0} & \multirow{2}{*}{1.36} \\
\hline & & $?$ & & & \\
\hline \multirow[t]{2}{*}{ PD-MeOAzo(PR) } & \multirow{2}{*}{ g } & 60 & \multirow{2}{*}{ I } & \multirow{2}{*}{63} & \multirow{2}{*}{1.24} \\
\hline & & $?$ & & & \\
\hline
\end{tabular}

${ }^{\mathrm{a}} \mathrm{PR}$, polymeric reaction. ${ }^{\mathrm{b}} \mathrm{g}$, glassy; $\mathrm{I}$, isotropic.

four polyurethane derivatives except PD-6-PP-MeO(PR) and PD-6-PP-CN, only one peak attributed to the melting point of the polyurethane derivatives was observed on the heating run, while in that for PD-6-PP-MeO(PR) and PD-6-PP-CN, no peak was shown on both the heating and cooling runs. These results indicated that no mesomorphic properties were exhibited for any of the polyurethane derivatives. In the case of the polyurethane derivatives with a methoxy group at the end of the mesogenic group, no mesomorphic properties were demonstrated with increasing flexible spacer length. As mentioned above, the PU-6-PP-MeO and $\mathrm{CN}$ showed a nematic phase. The PU-6-PP-CN is superior to the PU-6-PP-MeO with regard to its mesomorphic property owing to its mesomorphic temperature range of the cooling run. However the PD-6-PP-MeO and $\mathrm{CN}$ did not show any kinds of liquid crystalline phases. Regardless of whether the end group of the mesogen was a polar group or not, no mesomorphic properties for the polyurethane derivatives were observed. Smec- 
tic and nematic phases for the PU-MeOAzo were exhibited with a small decrease in the strength of the $\mathrm{H}$-bonding. However, no mesomorphic properties for the PD-MeOAzo were exhibited either. The substitution of methyl groups for hydrogen atoms in the urethane bonds interfered with the formation of $\mathrm{H}$-bonding for the rigid SLCPUs. This interference resulted in no mesomorphic properties for the polyurethane derivatives. From these results of thermal properties for the polyurethane derivatives, we concluded that the lateral interaction originating from $\mathrm{H}$-bonding played an important role in the exhibition of mesomorphic properties for the rigid SLCPUs.

\section{CONCLUSION}

The rigid SLCPUs exhibited mesomorphic properties, however from the results of thermally-controlled FT-IR measurements, the effect of $\mathrm{H}$-bonding on the mesomorphic properties for the rigid SLCPUs was not remarkable compared with that for flexible SLCPUs. In order to interfere with $\mathrm{H}$-bonding, we synthesized the polyurethane derivatives with methyl group substituted for hydrogen atom in the urethane bond. The polyurethane derivatives did not exhibit mesomorphic properties. Therefore, we concluded that the lateral interaction originating from $\mathrm{H}$-bonding played an important role in the exhibition of mesomorphic properties for the rigid SLCPUs.

\section{REFERENCES}

1. E. Akiyama and N. Koide, Liquid Crystals, 14, 1645 (1993).

2. T. Mihara, E. Akiyama, and N. Koide, Rep. Prog. Polym. Phys. Jpn., 36, 259 (1993)

3. N. Koide, E. Akiyama, and T. Mihara, Mol. Cryst. Liq. Cryst., 254, 283 (1994).

4. F. Papadimitrakopoulos, S. W. Kantor, and W. J. MacKnight, Polym. Prepr. Am. Chem. Soc. Div. Polym. Chem., 31(1), 486 (1990).

5. H. Finkelmann, M. Happ, M. Portugall, Makromol. Chem., 179, 2541 (1978)

6. T. Mihara and N. Koide, Rep. Progr. Polym. Phys. Jpn., 38, 203 (1995). 